\title{
Numerical solution for multi-term fractional delay differential equations
}

\author{
EMAN A. A. ZIADA ${ }^{a, *}$
}

a Nile Higher Institute for Engineering and Technology, Mansoura, Egypt

- Received: 20 September 2021 • Accepted: 05 December 2021 • Published Online: 30 December 2021

\begin{abstract}
In this paper, a multi-term nonlinear delay differential equation (DDE) of arbitrary order is studied. Adomian decomposition method (ADM) is used to solve these types of equations. Then the existence and stability of a unique solution will be proved. Convergence analysis of ADM is discussed. Moreover, the maximum absolute truncated error of Adomian's series solution is estimated. The stability of the solution is also discussed.
\end{abstract}

Keywords: Nonlinear delay differential equation, Arbitrary orders, Fixed point theorem, Convergence analysis, Stability, Adomian decomposition method.

\section{Introduction}

Differential equations of arbitrary orders and delay differential equations (DDEs) have many applications in engineering and science, including electrical networks, fluid flow, control theory, fractals theory, electromagnetic theory, viscoelasticity, potential theory, chemistry, biology, optical and neural network systems with delayed feedback [1][19]. In this paper, Adomian decomposition method (ADM) [20]-[26] is used to solve nonlinear fractional delay differential equations (FDDEs). This method has many advantages; it is efficiently works with different types of linear and nonlinear equations in deterministic or stochastic fields and gives an analytic solution for all these types of equations without linearization or discretization.

The paper is organized as follows: In section two ADM is applied to the problem under consideration. In section three uniqueness, convergence; error analysis and stability are discussed. Finally two numerical examples are presented by using MATHEMATICA package. 


\section{Formulation of the Problem}

Let $\alpha_{k} \in(n-1, n), k=1, \ldots, m$. Consider the nonlinear FDDE,

$$
\begin{aligned}
D^{n} x(t) & =f\left(t, x\left(t-r_{0}\right), D^{\alpha_{1}} x\left(t-r_{1}\right), \ldots, D^{\alpha_{m}} x\left(t-r_{m}\right)\right), t>0, \\
x^{(j)}(0) & =x_{0}^{j}, j=0,1, \ldots, n-1, \\
x(t) & =x_{0}, t \leqslant 0 .
\end{aligned}
$$

Where $D=\frac{d}{d t}, t \in I=[0, T], T \in R^{+}, x(t) \in C(I)$ and $D^{\alpha}$ is the Caputo derivative defined by,

$$
\begin{aligned}
D^{\alpha} x(t) & =I^{n-\alpha} D^{n} x(t), \quad n-1<\alpha \leqslant n, \\
I^{\alpha} x(t) & =\frac{1}{\Gamma(\alpha)} \int_{0}^{t}(t-\tau)^{\alpha-1} x(\tau) d \tau .
\end{aligned}
$$

Let

$$
D^{n} x(t)=y(t) \Rightarrow x(t)=\sum_{j=0}^{n-1} x_{0}^{j} \frac{t^{j}}{j !}+I^{n} y(t)
$$

Now

$$
\begin{aligned}
x(t) & =\sum_{j=0}^{n-1} x_{0}^{j} \frac{t^{j}}{j !}+\frac{1}{\Gamma(n)} \int_{0}^{t}(t-\tau)^{n-1} y(\tau) d \tau \Rightarrow \\
x(t-r) & =\sum_{j=0}^{n-1} x_{0}^{j} \frac{(t-r)^{j}}{j !}+\frac{1}{\Gamma(n)} \int_{0}^{t-r}(t-r-\tau)^{n-1} y(\tau) d \tau \\
& =\sum_{j=0}^{n-1} x_{0}^{j} \frac{(t-r)^{j}}{j !}+I_{r}^{n} y(t-r) .
\end{aligned}
$$

Then from equation (2.4), we get

$$
\begin{aligned}
D^{\alpha_{i}} x\left(t-r_{i}\right) & =I^{n-\alpha_{i}} D^{n} x\left(t-r_{i}\right) \\
& =I^{n-\alpha_{i}} y\left(t-r_{i}\right), \quad i=1,2, \ldots, m .
\end{aligned}
$$

Substituting the equations (2.4) and (2.5) into equation (2.1), we get

$$
y(t)=f\left(t, \sum_{j=0}^{n-1} x_{0}^{j} \frac{\left(t-r_{0}\right)^{j}}{j !}+I^{n} y\left(t-r_{0}\right), I^{n-\alpha_{1}} y\left(t-r_{1}\right), \ldots, I^{n-\alpha_{m}} y\left(t-r_{m}\right)\right) .
$$

Now, $f$ satisfies Lipschitz condition, i.e.,

$$
\left|f\left(t, y_{0}, y_{1}, \ldots, y_{m}\right)-f\left(t, z_{0}, z_{1}, \ldots, z_{m}\right)\right| \leqslant L \sum_{i=0}^{m}\left|y_{i}-z_{i}\right|,
$$


where $L$ is a Lipschitz constant, which implies that

$$
\begin{aligned}
& \mid f\left(t, \sum_{j=0}^{n-1} x_{0}^{j} \frac{\left(t-r_{0}\right)^{j}}{j !}+I^{n} y\left(t-r_{0}\right), \ldots, I^{n-\alpha_{m}} y\left(t-r_{m}\right)\right) \\
& -f\left(t, \sum_{j=0}^{n-1} x_{0}^{j} \frac{\left(t-r_{0}\right)^{j}}{j !}+I^{n} z\left(t-r_{0}\right), \ldots, I^{n-\alpha_{m}} z\left(t-r_{m}\right)\right) \mid \\
& \leqslant L \sum_{i=0}^{m}\left|I^{n-\alpha_{i}} y\left(t-r_{i}\right)-I^{n-\alpha_{i}} z\left(t-r_{i}\right)\right|, \quad \alpha_{0}=0 .
\end{aligned}
$$

The solution algorithm of equation (2.6) using ADM is,

$$
\begin{aligned}
& y_{0}(t)=p(t), \\
& y_{j}(t)=A_{j-1}(t), \quad j \geqslant 1 .
\end{aligned}
$$

Where $A_{j}$ are Adomian polynomials of the nonlinear term

$$
\begin{gathered}
f\left(t, \sum_{j=0}^{n-1} x_{0}^{j} \frac{\left(t-r_{0}\right)^{j}}{j !}+I^{n} y\left(t-r_{0}\right), \ldots, I^{n-\alpha_{m}} y\left(t-r_{m}\right)\right) \text { which take the form, } \\
A_{j}=\frac{1}{j !} \frac{d^{j}}{d \lambda^{j}}\left[f\left(t, \sum_{i=0}^{\infty} \lambda^{i} I^{n} y_{i}\left(t-r_{0}\right), \ldots, \sum_{i=0}^{\infty} \lambda^{i} I^{n-\alpha_{m}} y_{i}\left(t-r_{m}\right)\right)\right]_{\lambda=0} .
\end{gathered}
$$

Thus, the solution of equation (2.6) will be,

$$
y(t)=\sum_{i=0}^{\infty} y_{i}(t) .
$$

Finally,

$$
x(t)=\sum_{j=0}^{n-1} x_{0}^{j} \frac{t^{j}}{j !}+I^{n} y(t) .
$$

Remark: Let $0<\alpha_{1}<\alpha_{2}<\ldots<\alpha_{m}<n$. Then the nonlinear FDDE,

$$
\begin{aligned}
D^{n} x(t) & =f\left(t, x\left(t-r_{0}\right), D^{\alpha_{1}} x\left(t-r_{1}\right), \ldots, D^{\alpha_{m}} x\left(t-r_{m}\right)\right), t>0, \\
x^{(j)}(0) & =\left\{\begin{array}{l}
x_{0}, \quad j=0, \\
0, \quad j=1, \ldots, n-1,
\end{array}\right. \\
x(t) & =x_{0} \quad t \leqslant 0
\end{aligned}
$$

can be transformed to equation (2.6) and then applied the ADM.

\section{Analysis of Convergence}

\subsection{Uniqueness result}

Define $F: E \rightarrow E, E$ is the Banach space, $(C(I),\|\cdot\|)$, is the space of all continuous functions on I with the norm $\|x\|=\max _{t \in I} e^{-N t}|x(t)|, N>0$. 
Theorem 3.1. The problem (2.6) has one and only one solution $\mathrm{y}(\mathrm{t}) \in \mathrm{C}$ (I) if $\mathrm{f}$ satisfies the Lipschitz condition (2.7).

Proof. Define F : E $\rightarrow$ E as,

$$
F y(t)=f\left(t, \sum_{j=0}^{n-1} x_{0}^{j} \frac{\left(t-r_{0}\right)^{j}}{j !}+I^{n} y\left(t-r_{0}\right), I^{n-\alpha_{1}} y\left(t-r_{1}\right), \ldots, I^{n-\alpha_{m}} y\left(t-r_{m}\right)\right) .
$$

Let $y(t), z(t) \in E$, then

$$
\begin{aligned}
F y(t)-F z(t)= & f\left(t, \sum_{j=0}^{n-1} x_{0}^{j} \frac{\left(t-r_{0}\right)^{j}}{j !}+I^{n} y\left(t-r_{0}\right), \ldots, I^{n-\alpha_{m}} y\left(t-r_{m}\right)\right) \\
& -f\left(t, \sum_{j=0}^{n-1} x_{0}^{j} \frac{\left(t-r_{0}\right)^{j}}{j !}+I^{n} z\left(t-r_{0}\right), \ldots, I^{n-\alpha_{m}} z\left(t-r_{m}\right)\right)
\end{aligned}
$$

which implies that

$$
\begin{aligned}
& |F y(t)-F z(t)| \leqslant L \sum_{i=0}^{m}\left|I^{n-\alpha_{i}} y\left(t-r_{i}\right)-I^{n-\alpha_{i}} z\left(t-r_{i}\right)\right| \\
& \leqslant L \sum_{i=0}^{m}\left|\frac{1}{\Gamma\left(n-\alpha_{i}\right)} \int_{0}^{t}(t-\tau)^{n-\alpha_{i}-1}\left[y\left(\tau-r_{i}\right)-z\left(\tau-r_{i}\right)\right] d \tau\right| \\
& \operatorname{maxa}_{t \in I} e^{-N t}|F y(t)-F z(t)| \leqslant L \sum_{i=0}^{m} \frac{1}{\Gamma\left(n-\alpha_{i}\right)} \max _{t \in I} \int_{0}^{t} e^{-N(t-\tau)} \\
& \|F y-F z\| \leqslant L(t-\tau)^{n-\alpha_{i}-1} e^{-N \tau}\left|y\left(\tau-r_{i}\right)-z\left(\tau-r_{i}\right)\right| d \tau \\
& \leqslant L y-z \| \sum_{i=0}^{m} \frac{1}{\Gamma\left(n-\alpha_{i}\right)} \int_{0}^{\infty} e^{-N s} s^{n-\alpha_{i}-1} d s \\
& \sum_{i=0}^{m} \frac{1}{N^{n-\alpha_{i}}}\|y-z\| .
\end{aligned}
$$

Now, we choose $N$ large enough such that $L \sum_{i=0}^{m} \frac{1}{N^{n-\alpha_{i}}}<1$, we get

$$
\|\mathrm{Fy}-\mathrm{F} z\|<\|\mathrm{y}-z\|
$$

Then $F$ is a contraction and hence there exists one and only one solution $y(t) \in C$ (I) to the problem (2.6). 


\subsection{Convergence Proof}

Theorem 3.2. The series solution (2.10) of the problem (2.6) converges if $\left|\mathrm{y}_{1}(\mathrm{t})\right|<\mathrm{c}$, wherec is a positive constant.

Proof. Define the sequence $\left\{S_{p}(t)\right\}$ such that, $S_{p}(t)=\sum_{i=0}^{p} y_{i}(t)$ is the sequence of partial sums from the series solution $\sum_{i=0}^{\infty} y_{i}(t)$. From equations (2.8) and (2.9) we have,

$$
\sum_{i=0}^{\infty} y_{i}(t)=p(t)+\sum_{i=1}^{\infty} A_{i-1}(t)
$$

Let $S_{p}(t)$ and $S_{q}(t)$ are arbitrary partial sums, $p>q$, then

$$
S_{p}(t)=\sum_{i=0}^{p} y_{i}(t)=p(t)+\sum_{i=1}^{p} A_{i-1}(t) .
$$

And

$$
S_{q}(t)=\sum_{i=0}^{q} y_{i}(t)=p(t)+\sum_{i=1}^{q} A_{i-1}(t) .
$$

Now, we will prove that $\left\{S_{p}(t)\right\}$ is a Cauchy sequence in $E$.

$$
\begin{aligned}
& S_{p}(t)-S_{q}(t)=\sum_{i=1}^{p} A_{i-1}(t)-\sum_{i=1}^{q} A_{i-1}(t)=\sum_{i=q}^{p-1} A_{i}(t) \\
& \left|S_{p}(t)-S_{q}(t)\right| \leqslant L \sum_{i=0}^{m}\left|I^{n-\alpha_{i}} S_{p-1}\left(t-r_{i}\right)-I^{n-\alpha_{i}} S_{q-1}\left(t-r_{i}\right)\right| \\
& \leqslant L \sum_{i=0}^{m} \frac{1}{\Gamma\left(n-\alpha_{i}\right)} \int_{0}^{t}(t-\tau)^{n-\alpha_{i}-1}\left|S_{p-1}\left(\tau-r_{i}\right)-S_{q-1}\left(\tau-r_{i}\right)\right| d \tau \\
& e^{-N t}\left|S_{p}(t)-S_{q}(t)\right| \leqslant L \sum_{i=0}^{m} \frac{1}{\Gamma\left(n-\alpha_{i}\right)} \int_{0}^{t} e^{-N(t-\tau)}(t-\tau)^{n-\alpha_{i}-1} \\
& \times e^{-N \tau}\left|S_{p-1}\left(\tau-r_{i}\right)-S_{q-1}\left(\tau-r_{i}\right)\right| d \tau \\
& \left\|S_{p}-S_{q}\right\| \leqslant L \sum_{i=0}^{m} \frac{1}{N^{n-\alpha_{i}}}\left\|S_{p-1}-S_{q-1}\right\| \\
& \leqslant \beta\left\|S_{p-1}-S_{q-1}\right\| \text {. }
\end{aligned}
$$

Let $p=q+1$ then,

$$
\left\|S_{q+1}-S_{q}\right\| \leqslant \beta\left\|S_{q}-S_{q-1}\right\| \leqslant \beta^{2}\left\|S_{q-1}-S_{q-2}\right\| \leqslant \cdots \leqslant \beta^{q}\left\|S_{1}-S_{0}\right\| .
$$


From the triangle inequality we have,

$$
\begin{aligned}
\left\|S_{p}-S_{q}\right\| & \leqslant\left\|S_{q+1}-S_{q}\right\|+\left\|S_{q+2}-S_{q+1}\right\|+\cdots+\left\|S_{p}-S_{p-1}\right\| \\
& \leqslant\left[\beta^{q}+\beta^{q+1}+\cdots+\beta^{p-1}\right]\left\|S_{1}-S_{0}\right\| \\
& \leqslant \beta^{q}\left[\frac{1-\beta^{p-q}}{1-\beta}\right]\left\|y_{1}\right\| .
\end{aligned}
$$

Since, $0<\beta=L \sum_{i=0}^{m} \frac{1}{N^{n-\alpha_{i}}}<1$, and $p>q$ then, $\left(1-\beta^{p-q}\right) \leqslant 1$. Consequently,

$$
\begin{aligned}
\left\|S_{p}-S_{q}\right\| & \leqslant \frac{\beta^{q}}{1-\beta}\left\|y_{1}\right\| \\
& \leqslant \frac{\beta^{q}}{1-\beta} \max _{t \in I} e^{-N t}\left|y_{1}(t)\right|
\end{aligned}
$$

But, if $\left|y_{1}(t)\right|<c$ and as $q \rightarrow \infty$ then, $\left\|S_{p}-S_{q}\right\| \rightarrow 0$ and hence, $\left\{S_{p}(t)\right\}$ is a Cauchy sequence in $E$ so, the series $\sum_{i=0}^{\infty} y_{i}(t)$ converges.

\subsection{Analysis of Error}

Theorem 3.3. The maximum absolute truncation error of the solution (2.11) to the problem (2.1)- (2.3) is estimated by

Proof.

$$
\begin{aligned}
& \left\|x-\sum_{i=0}^{q} x_{i}\right\| \leqslant\left(\sum_{k=0}^{n-1} \frac{(-T)^{k}}{(k !) N^{n-k}}\right)\left(\frac{\beta^{q}}{1-\beta}\left\|y_{1}\right\|\right) \quad \text { if } n \text { is odd, } \\
& \text { and }\left\|x-\sum_{i=0}^{q} x_{i}\right\| \leqslant\left(\sum_{k=1}^{n-1} \frac{(-1)^{k+1}(T)^{k}}{\left(k ! N^{n-k}\right.}\right)\left(\frac{\beta^{q}}{1-\beta}\left\|y_{1}\right\|\right) \text { if } n \text { is even. }
\end{aligned}
$$

From Theorem 2 we have,

$$
\left\|S_{p}-S_{q}\right\| \leqslant \frac{\beta^{q}}{1-\beta} \max _{t \in I} e^{-N t}\left|y_{1}(t)\right|
$$

But, $S_{p}(t)=\sum_{i=0}^{p} y_{i}(t)$ as $p \rightarrow \infty$ then, $S_{p}(t) \rightarrow y(t)$ so,

$$
\left\|y-S_{q}\right\| \leqslant \frac{\beta^{q}}{1-\beta}\left\|y_{1}\right\|
$$

So,

$$
\left\|y-\sum_{i=0}^{q} y_{i}\right\| \leqslant \frac{\beta^{q}}{1-\beta}\left\|y_{1}\right\| .
$$

Form equation (2.11), we get

$$
\sum_{i=0}^{\infty} x_{i}(t)=\sum_{j=0}^{n-1} x_{0}^{j} \frac{t^{j}}{j !}+I^{n}\left(\sum_{i=0}^{\infty} y_{i}(t)\right) .
$$


Using equations (2.11) and (3.2), we obtain

$$
\begin{aligned}
& x(t)-\sum_{i=0}^{q} x_{i}(t)=I^{n} y(t)-I^{n}\left(\sum_{i=0}^{q} y_{i}(t)\right) \\
& =\int_{0}^{t} \cdots n-\text { fold } \cdots \int_{0}^{t}\left(y(\tau)-\sum_{i=0}^{q} y_{i}(\tau)\right) d \tau \cdots d \tau \text {. } \\
& e^{-N t}\left|x(t)-\sum_{i=0}^{q} x_{i}(t)\right|=\int_{0}^{t} \cdots n-\text { fold } \cdots \int_{0}^{t} e^{-N(t-\tau)} e^{-N \tau} \\
& \times\left|y(\tau)-\sum_{i=0}^{q} y_{i}(\tau)\right| d \tau \cdots d \tau \\
& \left\|x-\sum_{i=0}^{q} x_{i}\right\| \leqslant\left\|y-\sum_{i=0}^{q} y_{i}\right\| \int_{0}^{t} \cdots n-\text { fold } \cdots \int_{0}^{t} e^{-N(t-\tau)} d \tau \cdots d \tau .
\end{aligned}
$$

From equation (3.1), we get

$$
\left\|x-\sum_{i=0}^{q} x_{i}\right\| \leqslant\left(\sum_{k=0}^{n-1} \frac{(-T)^{k}}{(k !) N^{n-k}}\right)\left(\frac{\beta^{q}}{1-\beta}\left\|y_{1}\right\|\right) \quad \text { if } n \text { is odd, }
$$

and

$$
\left\|x-\sum_{i=0}^{q} x_{i}\right\| \leqslant\left(\sum_{k=1}^{n-1} \frac{(-1)^{k+1}(T)^{k}}{(k !) N^{n-k}}\right)\left(\frac{\beta^{q}}{1-\beta}\left\|y_{1}\right\|\right) \quad \text { if } n \text { is even. }
$$

And this completes the proof.

\subsection{Stability of the Solution}

Theorem 3.4. The solution of the problem (2.1)- (2.3) is uniformly stable.

Proof. Let $x(t)$ be a solution of the problem (2.1)- (2.3) and let $\widetilde{x}(t)$ be also a solution of this problem such that $\widetilde{x}^{(j)}(0)=\widetilde{x}_{0}^{j}$, then

$$
\begin{gathered}
|x(t)-\widetilde{x}(t)| \leqslant\left|\sum_{j=0}^{n-1} x_{0}^{j} \frac{t^{j}}{j !}-\sum_{j=0}^{n-1} \widetilde{x}_{0}^{j} \frac{t^{j}}{j !}\right|+\left|I^{n}[y(t)-\widetilde{y}(t)]\right| . \\
\|x-\widetilde{x}\| \leqslant \sum_{j=0}^{n-1}\left|x_{0}^{j}-\widetilde{x}_{0}^{j}\right| \frac{T^{j}}{j !}+\|y-\widetilde{y}\| \int_{0}^{t} \cdots n-\text { fold } \cdots \int_{0}^{t} e^{-N(t-\tau)} d \tau \cdots d \tau .
\end{gathered}
$$

From equations (3.3) and (3.4), we have

$$
\|x-\tilde{x}\| \leqslant \sum_{j=0}^{n-1} \frac{T^{j}}{j !}\left|x_{0}^{j}-\widetilde{x}_{0}^{j}\right|+\left(\sum_{k=0}^{n-1} \frac{(-T)^{k}}{(k !) N^{n-k}}\right)\|y-\widetilde{y}\| \text { if } n \text { is odd, }
$$


And

$$
\|x-\widetilde{x}\| \leqslant \sum_{j=0}^{n-1} \frac{T^{j}}{j !}\left|x_{0}^{j}-\widetilde{x}_{0}^{j}\right|+\left(\sum_{k=1}^{n-1} \frac{(-1)^{k+1}(T)^{k}}{(k !) N^{n-k}}\right)\|y-\widetilde{y}\| \text { if } n \text { is even. }
$$

From equation (2.6), we get

$$
\begin{aligned}
& |y(t)-\widetilde{y}(t)| \leqslant\left|\sum_{j=0}^{n-1} x_{0}^{j} \frac{\left(t-r_{0}\right)^{j}}{j !}-\sum_{j=0}^{n-1} \widetilde{x}_{0}^{j} \frac{\left(t-r_{0}\right)^{j}}{j !}\right|+ \\
& L \sum_{i=0}^{m}\left|I^{n-\alpha_{i}} y\left(t-r_{i}\right)-I^{n-\alpha_{i}} \widetilde{y}\left(t-r_{i}\right)\right| \\
& \leqslant \sum_{j=0}^{n-1}\left|x_{0}^{j}-\widetilde{x}_{0}^{j}\right| \frac{T^{j}}{j !}+ \\
& L \sum_{i=0}^{m} \frac{1}{\Gamma\left(n-\alpha_{i}\right)} \int_{0}^{t}(t-\tau)^{n-\alpha_{i}-1}\left|y\left(\tau-r_{i}\right)-\widetilde{y}\left(\tau-r_{i}\right)\right| d \tau \\
& \|y-\widetilde{y}\| \leqslant \sum_{j=0}^{n-1} \frac{T^{j}}{j !}\left|x_{0}^{j}-\widetilde{x}_{0}^{j}\right|+L \sum_{i=0}^{m} \frac{1}{N^{n-\alpha_{i}}}\|y-\widetilde{y}\| \\
& \|y-\widetilde{y}\| \leqslant\left(1-L \sum_{i=0}^{m} \frac{1}{N^{n-\alpha_{i}}}\right)^{-1}\left(\sum_{j=0}^{n-1} \frac{T^{j}}{j !}\left|x_{0}^{j}-\widetilde{x}_{0}^{j}\right|\right) .
\end{aligned}
$$

Substituting the equation (3.7) into equation (3.5), we get

$$
\|x-\tilde{x}\| \leqslant\left(1+\left(\sum_{k=0}^{n-1} \frac{(-T)^{k}}{(k !) N^{n-k}}\right)\left(1-L \sum_{i=0}^{m} \frac{1}{N^{n-\alpha_{i}}}\right)^{-1}\right)\left(\sum_{j=0}^{n-1} \frac{T^{j}}{j !}\left|x_{0}^{j}-\tilde{x}_{0}^{j}\right|\right) .
$$

Substituting the equation (3.7) into equation (3.6), we get

$$
\|x-\tilde{x}\| \leqslant\left(1+\left(\sum_{k=1}^{n-1} \frac{(-1)^{k+1}(T)^{k}}{(k !) N^{n-k}}\right)\left(1-L \sum_{i=0}^{m} \frac{1}{N^{n-\alpha_{i}}}\right)^{-1}\right)\left(\sum_{j=0}^{n-1} \frac{T^{j}}{j !}\left|x_{0}^{j}-\widetilde{x}_{0}^{j}\right|\right)
$$

if $n$ is even.

Therefore from equations (3.8) and (3.9), if $\sum_{j=0}^{n-1} \frac{T^{j}}{j !}\left|x_{0}^{j}-\widetilde{x}_{0}^{j}\right|<\delta(\epsilon)$, then $\|x-\tilde{x}\|<\epsilon$, which completes the proof. 


\section{Numerical Method and Results}

Example 4.1. Consider the following nonlinear FDDE,

$$
\begin{gathered}
\frac{d x(t)}{d t}=\frac{1}{2}(1-t)+\frac{1}{5} x(t-0.3)+\frac{1}{15}\left(D^{1 / 3} x(t-0.05)\right)^{3}+\frac{1}{20}\left(D^{1 / 4} x(t-0.4)\right)^{4}, \quad t>0 \\
x(t)=0.1, \quad t \leqslant 0 .
\end{gathered}
$$

Using equations (2.4)-(2.6), we get

$$
y(t)=\left(0.52-\frac{t}{2}\right)+\frac{1}{5}[I y(t-0.3)]+\frac{1}{15}\left[I^{2 / 3} y(t-0.05)\right]^{3}+\frac{1}{20}\left[I^{3 / 4} y(t-0.4)\right]^{4}
$$

Applying ADM to equation (4.2), we have

$$
\begin{aligned}
& y_{0}(t)=\left(0.52-\frac{t}{2}\right), \\
& y_{i}(t)=\frac{1}{5}\left[I_{i-1}(t-0.3)\right]+\frac{1}{15} A_{i-1}(t)+\frac{1}{20} B_{i-1}(t), \quad i \geqslant 1 .
\end{aligned}
$$

Where $A_{i}$ and $B_{i}$ are Adomian polynomials of the nonlinear terms $\left[I^{2 / 3} y(t-0.05)\right]^{3}$ and $\left[\mathrm{I}^{3 / 4} \mathrm{y}(\mathrm{t}-0.4)\right]^{4}$ respectively. From equations (4.3)and (4.4), the solution of the problem (4.2) is,

$$
y(t)=\sum_{i=0}^{m} y_{i}(t) .
$$

Finally

$$
\begin{aligned}
x(t)= & 0.1+I[y(t)] \\
= & 0.1+0.52 t-0.18697 t^{2}-0.00732539 t^{3}-0.00130407 t^{4} \\
& -0.00206309 t^{5}+0.00117699 t^{6}-0.000528417 t^{7}+\cdots .
\end{aligned}
$$

The maximum absolute truncated error at different values of $m$ (when $t=1, N=10$ ) are given in Table 1. Figure 1 shows ADM solution (when $m=5$ ).

Table 1: Max. Absolute Error
\begin{tabular}{|c|c|}
\hline $\mathrm{m}$ & Max. error \\
\hline 5 & $3.07849 \times 10^{-8}$ \\
\hline 10 & $9.93029 \times 10^{-14}$ \\
\hline 15 & $3.20321 \times 10^{-19}$ \\
\hline 20 & $1.03326 \times 10^{-24}$ \\
\hline
\end{tabular}

Example 4.2. Consider the following nonlinear FDDE,

$$
D^{2} \chi(t)=2-\frac{2 t}{\pi}\left(\frac{2 t}{3}-0.1\right)^{2}-\frac{64 t^{2}}{5 \pi^{2}}+\frac{1}{8}\left(D^{1 / 2} \chi(t-0.1)\right)^{2}+\frac{1}{20}\left(D^{3 / 2} \chi(t-0.2)\right)^{4}, t>0,
$$




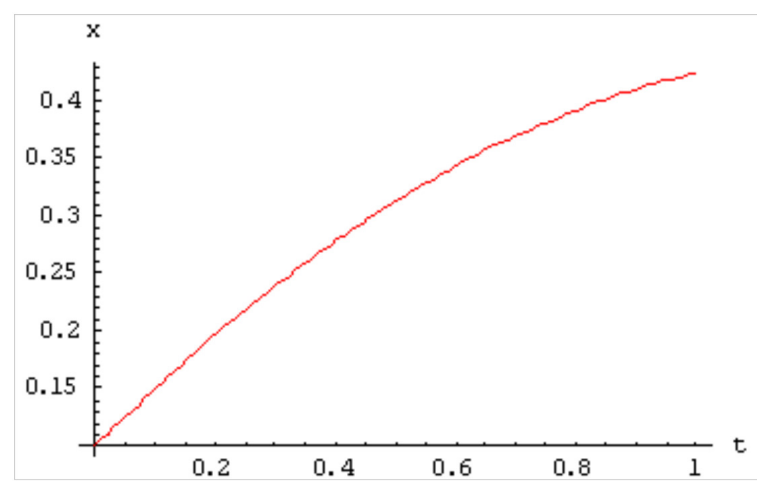

Figure 1: ADM solution.

$$
\begin{aligned}
& x(0)=0, x^{\prime}(0)=0, \\
& x(t)=0, \quad t \leqslant 0,
\end{aligned}
$$

The exact solution of this problem is $\mathrm{t}^{2}$.

By using equations (2.4)-(2.6), we get

$$
y(t)=2-\frac{2 t}{\pi}\left(\frac{2 t}{3}-0.1\right)^{2}-\frac{64 t^{2}}{5 \pi^{2}}+\frac{1}{8}\left[I^{3 / 2} y(t-0.1)\right]^{2}+\frac{1}{20}\left[I^{1 / 2} y(t-0.2)\right]^{4}
$$

Where $x^{\prime}(0)=0$.

Applying ADM to equation (4.7), we have

$$
\begin{aligned}
& y_{0}(t)=2-\frac{2 t}{\pi}\left(\frac{2 t}{3}-0.1\right)^{2}-\frac{64 t^{2}}{5 \pi^{2}} \\
& y_{i}(t)=\frac{1}{8} A_{i-1}(t)+\frac{1}{20} B_{i-1}(t), \quad i \geqslant 1 .
\end{aligned}
$$

Where $A_{i}$ and $B_{i}$ are Adomian polynomials of the nonlinear terms $\left[I^{3 / 2} y(t-0.1)\right]^{2}$ and $\left[I^{1 / 2} y(t-0.2)\right]^{4}$. From equations (4.8) and (4.9), the solution of the problem (4.7) is,

$$
y(t)=\sum_{i=0}^{q} y_{i}(t) .
$$

Finally

$$
x(t)=I^{2}[y(t)] .
$$

The absolute error of ADM series solution (when $q=2$ ) are shown in Table 2 and the maximum absolute truncated error (using Theorem 3) at different values of $q$ (when $\mathrm{t}=1, \mathrm{~N}=25$ ) are given in Table 3. Figure 2 shows ADM and exact solutions (when $q=2)$. 


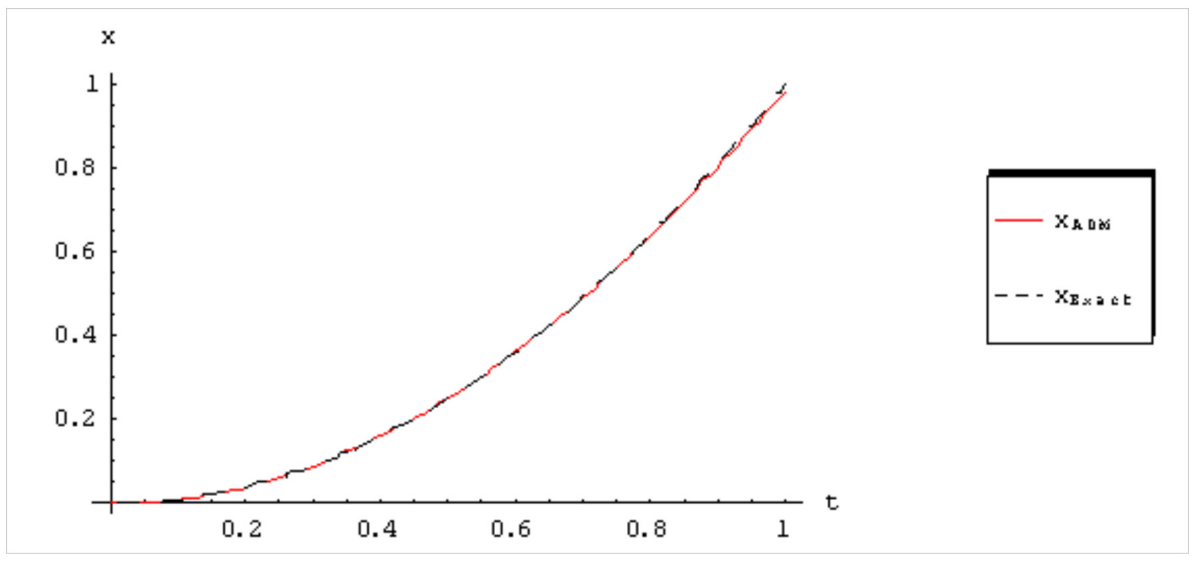

Figure 2: ADM and exact solutions.

Table 2: Absolute Error

\begin{tabular}{|c|c|}
\hline$t$ & $\left\|x_{\text {Exact }}-x_{\text {ADM }}\right\|$ \\
\hline 0.1 & $9.75482 \times 10^{-7}$ \\
\hline 0.2 & $3.49447 \times 10^{-6}$ \\
\hline 0.3 & $6.8164 \times 10^{-6}$ \\
\hline 0.4 & 0.0000487299 \\
\hline 0.5 & 0.0000934568 \\
\hline 0.6 & 0.0000266693 \\
\hline 0.7 & 0.000740444 \\
\hline 0.8 & 0.00287597 \\
\hline 0.9 & 0.0077946 \\
\hline 1 & 0.0174979 \\
\hline
\end{tabular}

Table 3: Max. Absolute Error

\begin{tabular}{|l|l|}
\hline $\mathrm{q}$ & Max. error \\
\hline 2 & 0.02614538 \\
\hline 5 & 0.00574685 \\
\hline 10 & 0.000460068 \\
\hline 15 & 0.0000368312 \\
\hline 20 & $2.94855 \times 10^{-6}$ \\
\hline
\end{tabular}

\section{Conclusion}

In this paper, an interesting method (ADM) has been used to solve a nonlinear multiterm fractional delay differential equation. This method has given an analytical solution. Moreover, when we have compared the ADM solution with the exact solution, we have seen that it gives a good approximate solution and it is enclosed with the results obtained from using Theorem 3 (see Tables 1,3).

\section{References}

[1] Chinnathambi R, Rihan FA, Alsakaji HJ (2021). A fractional-order model with time delay for tuberculosis with endogenous reactivation and exogenous reinfections. Mathematical methods in the applied sciences, 44(10): 8011-8025. https://doi.org/10.1002/mma.5676.

[2] Rihan FA, Al-Mdallal QM, AlSakaji HJ, Hashish A (2019). A fractional-order epidemic model with timedelay and nonlinear incidence rate. Chaos, Solitons and Fractals, 126: 97-105. https:/DOI:10.1016/j. chaos.2019.05.039. 
[3] Alam Khan N, Abdul Razzaq O, Ara A, Riaz F (2016). Numerical Solution of System of Fractional Differential Equations in Imprecise Environment. In: Lepez-Ruiz, R., Ed., Numerical Simulation: From Brain Imaging to Turbulent Flows, IntechOpen, London, 167-186. https://doi .org/10.5772/64150.

[4] Atangana A, Alabaraoye E (2013). Solving a system of fractional partial differential equations arising in the model of HIV infection of CD4+ cells and attractor one-dimensional Keller-Segel equations. Advances in Difference Equations, 94: 1-14. https://doi.org/10.1186/1687-1847-2013-94.

[5] Hammad HA, De la Sen M (2021). Tripled fixed point techniques for solving system of tripled-fractional differential equations. AIMS Mathematics, 6(3): 2330-2343. https://doi .org/10.3934/math. 2021141.

[6] Rida SZ, Arafa AAM (2011). New Method for Solving Linear Fractional Differential Equations, International Journal of Differential Equations, 1-8. https://doi .org/10.1155/2011/814132.

[7] Daraghmeh A, Qatanani N, Saadeh A (2020). Numerical Solution of Fractional Differen-tial Equations. Applied Mathematics, 11: 1100-1115. https://doi.org/10.4236/am. 2020.1111074.

[8] Miller KS, Ross B (1993). An Introduction to the Fractional Calculus and Fractional Differential Equations. Wiley-Interscience, New York.

[9] Podlubny I (1999). Fractional Differential Equations. Academic Press, New York.

[10] Kilbas AA, Srivastava HM, Trujillo JJ (2006). Theory and Applications of Fractional differential equations. Elsevier, New York.

[11] Abd El-Salam Sh A, El-Sayed AMA (2007). On the stability of some fractional-order non-autonomous systems, Electronic Journal of Qualitative Theory of Differential Equations, 6: 1-14. https://doi .org/10. 14232/ejqtde.2007.1.6.

[12] El-Sayed AMA, Abd El-Salam Sh A (2008). On the stability of a fractional-order differential equation with nonlocal initial condition. Electronic Journal of Qualitative Theory of Differential Equations, 29: 1-8. https://doi.org/10.14232/ejqtde.2008.1.29.

[13] Evans DJ, Raslan KR (2005). The Adomian decomposition method for solving delay differential equation. International Journal of Computer Mathematics, (UK), 82: 49-54. https://doi.org/10.1080/ 00207160412331286815.

[14] Zwillinger D (1997). Handbook of Differential Equations, Academic Press.

[15] Mensour B, Longtin A (1998). Chaos control in multistable delay-differential equations and their singular limit maps. Pysical Review E, 58: 410-422. https://doi.org/10.1103/PhysRevE. 58.410.

[16] Hefferan JM, Corless RM (2005). Solving some delay differential equations with computer algebra. Applied Probability Trust, 1-22.

[17] El-Sayed AMA, El-Mesiry EM, El-Saka HAA (2004). Numerical solution for multi-term fractional (arbitrary) orders differential equations. Comput. and Appl. Math., 23(1): 33-54.

[18] El-Mesiry EM, El-Sayed AMA, El-Saka HAA (2005). Numerical methods for multi-term fractional (arbitrary) orders differential equations. Appl. Math. and Comput., 160(3): 683-699. https://doi.org/10. $1016 / j$.amc. 2003.11.026.

[19] Al-Mdallal QM, Hajji MA, Abdeljawad T (2021). On the iterative methods for solving fractional initial value problems. Journal of Fractional Calculus and Nonlinear Systems, 2(1): 76-81. https://doi .org/ 10.48185/jfcns.v2i1.297.

[20] Adomian G (1994). Solving Frontier Problems of Physics: The Decomposition Method. Kluwer, https: //doi.org/10.1007/978-94-015-8289-6.

[21] Adomian G (1983). Stochastic System. Academic press.

[22] Adomian G (1986). Nonlinear Stochastic Operator Equations. Academic press, San Diego. https://doi. org/10.1016/C2013-0-10271-1.

[23] Adomian G (1989). Nonlinear Stochastic Systems: Theory and Applications to Physics. Kluwer.

[24] Abbaoui K, Cherruault Y (1994). Convergence of Adomian's method applied to differential equations, Computers Math. Applic., 28: 103-109. https://doi.org/10.1016/0898-1221(94)00144-8.

[25] Cherruault Y, Adomian G, Abbaoui K, Rach R (1995). Further remarks on convergence of decomposition method. International J. of Bio-Medical Computing., 38: 89-93. https://doi.org/10.1016/ 0020-7101 (94)01042-Y.

[26] Shawaghfeh NT (2002). Analytical approximate solution for nonlinear fractional differential equations. J. Appl. Math. Comput., 131: 517-529. https://doi .org/10.1016/S0096-3003(01) 00167-9. 